https://helda.helsinki.fi

Early lexical development of Finnish children: A longitudinal study

Stolt, Suvi

2008

Stolt , S , Haataja , L , Lapinleimu , H \& Lehtonen , L 2008 , ' Early lexical development of

pÿFinnish children: A longitudinal study ' , First Language , vol. 28 , no. 3 , pp. 259279 . https://doi.org/10.1177/0142

http://hdl.handle.net/10138/309067

https://doi.org/10.1177/0142723708091051

publishedVersion

Downloaded from Helda, University of Helsinki institutional repository.

This is an electronic reprint of the original article.

This reprint may differ from the original in pagination and typographic detail.

Please cite the original version. 


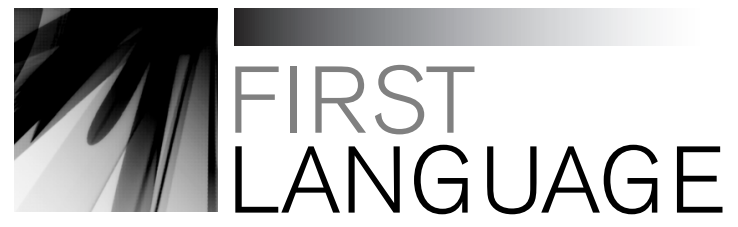

\title{
Early lexical development of Finnish children: A longitudinal study
}

\author{
Suvi Stolt, University of Helsinki and Turku University Central Hospital \\ Leena Haataja, Turku University Central Hospital \\ Helena Lapinleimu, Turku University Central Hospital \\ Liisa Lehtonen, Turku University Central Hospital
}

\begin{abstract}
The growth rate and the development of the composition of the receptive and expressive lexicon were studied in a longitudinal sample of 35 Finnish children. The MacArthur Communicative Development Inventory was used to gather data of the receptive lexicon at $0 ; 9,1 ; 0$ and $1 ; 3$, and the expressive lexicon at $0 ; 9,1 ; 0,1 ; 3,1 ; 6$ and 2;0. The receptive lexicon was acquired earlier, at a faster rate and with higher individual variation than the expressive lexicon. A gender difference was found in expressive vocabulary, but not in receptive vocabulary. The growth trajectories of semantic lexical categories detected in both lexicons resembled each other. Verbs were acquired more readily in receptive lexicons. Results support a universal sequence in the development of the composition of the lexicon.
\end{abstract}

\section{KEYWORDS}

Acquisition of Finnish; expressive lexicon; individual differences; longitudinal study; MacArthur CDI; receptive lexicon; vocabulary spurt

\section{INTRODUCTION}

The acquisition of the first lexicon is one of the central issues in the field of child language research. Most studies have concentrated on vocabulary development in 
English children. In contrast, the number of the studies focusing on lexical acquisition of children acquiring other languages is smaller (see, e.g., Bassano, Eme \& Champaud, 2005; D'Odorico, Carubbi, Salerni \& Calvo, 2001; Eriksson \& Berglund, 1999; Kern, 2007; Salerni, Assanelli, D’Odorico \& Rossi, 2007; Szagun, Steinbrink, Franik, \& Stumper, 2006; Thordardottir \& Weismer, 1996). A crosslinguistic perspective is needed to understand fully how children acquire the competence in their native language. The aim of the present study is to provide a description of the growth rate, and the development of the composition of early receptive vocabulary between 0;9 and $1 ; 3$, and the expressive lexicon between $0 ; 9$ and 2;0 in Finnish children.

\section{The growth of the early lexicon}

Children show the first signs of word comprehension before their first birthday. In a large cross-sectional normative study of the vocabulary checklist method, the MacArthur Communicative Development Inventory (CDI; Fenson et al., 1994), in which lexical data of over 1800 children acquiring English aged between 8 and 30 months were analysed, the mean value of the receptive lexicon size was 36 (median 17) words at 0;8, 67 (median 41) words at 0;10, and 191 (median 169) words at 1;4. There was a difference between mean and median values at each age point suggesting variability in the onset and growth rate in the early receptive lexicon (Bates, Dale \& Thal, 1995). The first words are produced at around 1;0, and the expressive lexicon is acquired slowly during the first months of the second year. In the normative CDI study the mean expressive lexicon size was 10 words (median 6) at 1;0, and 64 words (median 40) at $1 ; 4$. At 2;0 the mean vocabulary size was 312 words (standard deviation 1.28; range 89-534 words; Bates et al., 1995). Studies contrasting the acquisition rates of receptive and expressive vocabularies suggest that the receptive lexicon is not only acquired earlier, but also at a faster rate than expressive vocabulary. Benedict (1979) analysed the acquisition of the first 50 comprehended and produced words of eight children using a combination of checklist and diary methods, and reported that the mean acquisition age interval from 10 to 50 words was 2.7 months in the receptive lexicon, while in the expressive lexicon it was 4.8 months. The earlier and faster acquisition of receptive vocabulary was also found in a follow-up study of 58 children reported by Menyuk, Liebergott \& Schultz (1995), and in a large crosslinguistic study of children acquiring English and Italian (Caselli et al., 1995).

One issue often apparent in the acquisition of the expressive lexicon is a rapid acceleration in the growth rate called 'vocabulary spurt' or 'vocabulary burst' usually reported to occur when children have acquired roughly 20-40 words in their expressive vocabulary (e.g., Barrett, 1995). The definition used for spurt in different studies varies. For example, Goldfield \& Reznick (1990) used a criterion of the acquisition of 10 new words for each 21/2-week interval, and Mervis \& Bertrand (1995) the criterion of the acquisition of 10 new words, with at least five of them object words, in a 2-week interval. Barrett (1995) defined a spurt as a sudden acceleration of lexicon acquisition rate, during which a child acquires at least eight new words in a week within 2 or 3 weeks. A vocabulary spurt has not been found in all children, however, and there has been discussion whether the spurt actually exists, whether it occurs in considerably fewer children than often thought, or whether it happens 
later in some children than in others (Bates et al., 1995; Dromi, 1999; Ganger \& Brent, 2004; Goldfield \& Reznick, 1990, 1996; Mervis \& Bertrand, 1995; Tomasello, 2003). This acceleration in the growth rate of the expressive lexicon may be best described as smoothly accelerating with no single 'take-off point' as assumed by the definitions of spurt (Bates et al., 1995). Whether there exists a spurt-like acceleration in the acquisition of the receptive lexicon is even less clear. Reznick \& Goldfield (1992) reported, however, that their data revealed a spurt in comprehension scores for some but not all children mostly between $1 ; 8$ and 1;10. The comprehension spurt was associated with the presence of the production spurt.

Girls as a group tend to acquire their lexicons earlier than boys (e.g., Bornstein, Leach \& Haynes, 2004; Fenson et al., 1994). This difference in acquisition has been found in both the receptive and expressive vocabulary (Fenson et al., 1994). However, this advantage is small compared with the large intra-gender variability in early lexical acquisition (Fenson et al., 1994). One reason for the sex-based difference may be in maturation that affects the growth of verbal capacity (Bornstein, Haynes \& Painter, 1998).

\section{The development of the composition of the lexicon}

Children acquire words belonging to different semantic categories of adult language from early on (Tomasello, 2003). However, it seems that nouns are acquired more readily than verbs or other words in different languages (Bornstein et al., 2004; Gentner, 1982). This is interesting because languages differ in the way nouns and verbs are used: in some languages nominal words have to be in all clauses, and therefore nouns are more salient in the speech stream, whereas in other languages it is possible to use clauses that consist of verbs only (Tomasello, 2003). Gentner explained the noun-dominance in early lexical acquisition by hypothesizing (1982) that nouns are acquired earlier than verbs because they refer to concrete objects, and thus the mapping between language and conceptual world is more transparent in nouns than in verbs which refer to actions and events. Gentner \& Boroditsky (2001) have hypothesized further that words differ in how they take their meaning in relation to context, i.e., entities of the world versus linguistic connections in a clause. The primary function of nouns and other open-class words is to denote perceptual entities in the world, whereas relational words such as closedclass words take their meaning from the linguistic connections among the referential words, and are thus more linguistically dominant than nouns. Verbs are in the middle of this continuum: although they refer to the context, they also rely on their arguments to denote their meaning fully (Gentner \& Boroditsky, 2001; Tomasello, 2003).

Based on their analyses of children acquiring English and Italian (Bates, Bretherton \& Snyder, 1988; Bates et al., 1994; Caselli et al., 1995; Caselli, Casadio \& Bates, 1999), Bates and Caselli have expanded Gentner's noun-verb sequence hypothesis. According to their proposal, the first produced words are often connected to routine situations or language games. These first words are not necessarily object names, but onomatopoetic expressions, and names of people are acquired as well. These early lexical items are used in varying ways, and they are difficult to classify 
under any semantic category of adult language. When the lexicon size is between 50 and 200 words, the majority of the words are nouns. The growth in the number and proportional share of nouns can be interpreted to reflect that the child is establishing the reference. Predication, the ability to encode relational meanings, cannot emerge before children have acquired a sufficient number of content words, and thus verbs and adjectives are rare in small lexicons (Caselli et al., 1995; Caselli et al., 1999). Verb acquisition in English children starts only after the lexicon size has exceeded 100-200 words.

Grammatical function words (i.e., prepositions, pronouns, question words, quantifiers, connectives) are very rare in early lexicons, and these are acquired at an even slower rate than verbs. The proportional growth of function words starts when children have acquired a total vocabulary size of 300-500 words. The growth of the function words reflects a developmental shift to the acquisition of grammar (Bates et al., 1994; Caselli et al., 1995; Caselli et al., 1999). This developmental shift from routines to reference to predication and to grammar is based on cognitive growth, for example, on the growth of the perceptual acuity and/or memory skills (Bates et al., 1994). It reflects universal developments in the logical and conceptual substrates of meaning (Caselli et al., 1999). The parallel developmental path in the acquisition of early lexicon as proposed by Bates and Caselli has been reported in Spanish (Jackson-Maldonado, Thal, Marchman, Bates \& Gutierrez-Clellen, 1993), English and Italian (Caselli et al., 1995; Caselli et al., 1999) and Hebrew (Maital, Dromi, Sagi \& Bornstein, 2000) (compare Bornstein, Cote, Maital, Painter, Park \& Pascual, 2004; Kauschke \& Hofmeister, 2002).

There are only a few studies comparing the acquisition of the semantic word classes in the receptive and expressive lexicon. They propose that a similar kind of developmental shift from routine words to closed-class words can be detected in the receptive lexicon as found in the expressive lexicon. However, it seems that verbs are acquired faster in comprehension vocabularies than in the expressive lexicons (Benedict, 1979; Menyuk et al., 1995). Caselli et al. (1995) analysed the composition of both receptive and expressive vocabularies in samples of 659 English and 195 Italian children gathered between 0;8 and 1;4 using the MacArthur Communicative Development Inventory (CDI; Fenson et al., 1994), and reported that the acquisition order of the words belonging to different adult language categories was highly parallel in both vocabularies in both languages. The acquisition of verbs began earlier for the receptive vocabulary (Caselli et al., 1995). In addition, the findings of Harris, Yeeles, Chasin \& Oakley (1995) suggested other kinds of symmetries between comprehension and production lexicons: contextually flexible words in production tended to be so in comprehension, and words that were context-bound were so in both modalities.

\section{Lexical acquisition of Finnish children}

There are some studies focusing on the growth of early lexicon in Finnish children. Kunnari (2000) followed the growth of the expressive lexicons of 10 children using maternal diaries, from the acquisition of the first word to a lexicon size of 50 words. The mean age at which children produced their first words was 11 months 
and 5 days (range 8.9-13.9), and the mean age for the acquisition of 50 words was 17 months and 7 days (13.2-22.7). All children demonstrated a spurt according to the definition of Barrett (1995), most of them when the expressive lexicon size was from 20 to 30 words (Kunnari, 2000). Nieminen (1991) followed the growth of early receptive and expressive vocabularies of 10 children, and found that they acquired receptive lexicons four to five times faster than expressive lexicons. Furthermore, in the standardization study of the Finnish version of the MacArthur Communicative Development Inventory (Lyytinen, 1999) the mean value of the comprehended words of 95 full-term healthy children was 89 words (SD 63, range $6-263)$ at $1 ; 0$, and 164 words (SD 83, range 16-322) at $1 ; 2$. Respectively, the mean value of the expressive lexicon size was 7 words (SD 9, range $0-60)$ at 1;0, 70 words (SD 78, range $0-384$ ) at $1 ; 6$, and 278 words (SD 163, range $0-595$ ) at 2;0. In addition, in our recent study (Stolt et al., 2007) we found that the lexicon size of 84 two-year-old cognitively normally developing full-term Finnish children measured using the Finnish version of the CDI was highly comparable (272 words, SD 161) to that presented by Lyytinen (1999).

Studies focusing on the development of vocabulary composition in Finnish children are rare. In the follow-up study of Nieminen (1991), the first understood words were names of people, usually object names (i.e., toys, animals, household items, food), and verbs describing concrete actions. The first produced words were very much the same as those understood. Nieminen did not analyse the composition of the early lexicon in relation to lexicon size. In our earlier study (Stolt et al., 2007) the composition of the lexicon of 84 children at 2;0 was analysed in relation to vocabulary size, and the compositions were comparable to the proposal of Bates et al. (1994) and Caselli et al. (1999). This study provided information on the lexical composition of Finnish children at 2;0. Until now the development of the composition of early receptive and expressive lexicons in relation to lexical growth during the second year has not been studied in Finnish children.

\section{Aspects of Finnish}

Finnish is an agglutinative language in which grammatical relations are expressed mainly using suffixes (Dasinger, 1997). The morphological system is extensive. Nominal words (i.e., nouns, adjectives, numerals and pronouns) are inflected via 15 cases. In the verb inflection system there is a subject-verb agreement. In addition, verbal morphology for finite verbs can express voice (active, passive), mood (indicative, imperative, conditional, potential), and tense (past, non-past). Because of the intensive use of suffixes, Finnish words are relatively long. This lengthiness may affect the auditory discrimination of words (Lyytinen \& Lyytinen, 2004). However, the different kinds of suffix added to the word stem may also help children in the categorization process when figuring out how words are used, for example as a noun or as a verb.

\section{Aims of the study}

The present study had two goals. The first was to obtain information on the growth of early receptive and expressive lexicons. The purpose was to analyse and describe 
the growth rates of both lexicons, and to study the effect of gender on the growth of both vocabularies. The second was to study the development of the compositions of the receptive and expressive lexicons in Finnish children. We wanted to study whether the kinds of growth trajectories of semantic word categories which have been identified in the literature on development in other languages could be detected in the lexicons of Finnish children.

\section{METHOD}

\section{Participants}

The participants were 35 (18 boys, 17 girls) full-term children. All were singletons, their mothers' firstborns, and were children of monolingual Finnish-speaking families. The children were born between November 2001 and April 2002 in Turku University Central Hospital. The invitation to the study was presented to all families, 80 altogether, in the maternity ward having a baby fulfilling the inclusion criteria presented above. Forty-four families (55\%) agreed to participate, and the language development of 35 children was followed up to 2;0 years of age. In the present study, data concerning lexical development are presented. The children formed a control group in a multidisciplinary follow-up study focusing on the development of prematurely born very-low-birth-weight children (PIPARI study).

The language skills of the children were developing within normal expectations. The mean value of the group on the Reynell Developmental Language Scales III (Edwards et al., 1997; Kortesmaa, Merikoski, Warma \& Varpela, 2001) at 2;0 was 109 (SD 13, range 83-132). One child had a standard score $<85$ in Reynell's test, and was sent to the audiologist who verified normal hearing ability. The children grew up in middle- or upper middle-class families: the length of the mothers' basic education was 9-12 years in 9 children (26\%), and over 12 years in 26 children (74\%).

\section{Method and data collection}

The standardized Finnish version of the MacArthur Communicative Development Inventory (Fenson et al., 1994; Lyytinen, 1999) was used to collect data on lexical development. Information on receptive lexicon was gathered at three age points, $0 ; 9,1 ; 0$ and 1;3, using the CDI Infant form: Words and Gestures. Data of the expressive lexicon were collected at five age points, 0;9, 1;0 and 1;3, using the CDI Infant form, and at 1;6 and 2;0 using the CDI Toddler Form: Words and Sentences. In the infant form of the Finnish version of CDI there are 380 items presented in 19 semantic categories. The vocabulary checklist of the Words and Sentences form consists of 595 items presented in 20 semantic categories. Good reliability for the English version of the CDI has been demonstrated (e.g., Fenson et al., 1994; Ring \& Fenson, 2000), and for the Finnish version (e.g., Lyytinen, 1999). In the normative study of the Finnish version of the CDI, the correlation coefficient between the receptive lexicon size at 1;2 and the receptive score in Reynell Developmental Language Scales at $1 ; 6$ was good $(r=0.43, p=0.001)$, and the concurrent correlation 
between the expressive lexicon size in CDI and the expressive scale of Reynell Developmental Language Scales at 1;6 was excellent, $r=0.85, p=0.0001$ (Lyytinen, 1999).

Parents were encouraged to give as much information as possible about their child's lexical development. When filling in the CDI form they were asked to write down all the words a child was using as accurately as possible in order to get suggestive information about the phonological representation of the word. Parents were also asked to write down all the words the child used, but were not included in the CDI lists. Furthermore, in order to obtain information about the lexical development that was as valid as possible, the criteria for the comprehended and expressed words were established. A word was accepted as understood if a child showed repeatedly a clear, immediate and correct response to it (Benedict, 1979). The criteria of the expressed word used in the present study and specified in the Finnish form of the CDI are: (1) spontaneous, not only imitated, use of the word; (2) the word is connected repeatedly to the same referent. These criteria were established at the appointment before the collection of vocabulary data began when the child was 0;6. Mother-child pairs were met at each of the five age points when the lexical data were gathered. During these appointments the CDI form was reviewed with the mother to verify that the criteria for a word were met.

\section{Analysis and statistics}

The words marked in the CDI form and all reported extra words were accepted only once. Extra words were classified according to the sections of CDI (e.g., extra verbs were classified under the category of action words, or adjectives under the category of descriptive words). The checklist and extra words were counted separately. In the analyses, the combined value was used including both checklist and extra words because the aim was to obtain a detailed picture of lexical acquisition during the second year. Although checklist methods have been shown to be reliable and valid (e.g., Bates et al., 1994; Fenson et al., 1994), the purpose of these methods is not to capture all the words in children's lexicons, but to be a representative index of early vocabularies (Bornstein, Putnick \& De Houwer, 2006). It has also been shown that checklist methods may underestimate the vocabulary size in large lexicons (Robinson \& Mervis, 1999). Using the combination of checklist and diary method made it possible to take into consideration also those words not included in the CDI wordlist and thus to get a more comprehensive picture of children's lexicons (see also Benedict, 1979).

To analyse the growth rates of the receptive and expressive lexicons, the number of acquired new words in both lexicons in each data collection interval was calculated from the combined values for each child and for the group. In addition, the value often used in the studies focusing on the vocabulary spurt (10 new words in 2-week interval; e.g., D'Odorico et al., 2001; Mervis \& Bertrand, 1995) was applied: the value of the acquisition of 60 new words or more per each 3-month period, and 120 new words for the 6 -month interval from $1 ; 6$ to $2 ; 0$ was used. We want to emphasize, however, that because of the long intervals between the data collection points the aim of the present study was not to analyse the vocabulary spurt itself. The value was 
used to obtain information on the possible change in the growth rate and to make rough comparisons between the findings of the present study and the earlier ones.

The purpose of the composition analysis was to get a picture of the development of the lexical composition in relation to age, and in relation to the lexicon size. The combined lexical values were used. The compositions of the receptive and expressive lexicons of the group at different age points were analysed first. Then, the compositions of the receptive and expressive lexicons of different sizes were described. To do this, all gathered lexicons were arranged in groups according to size. For receptive lexicons, all children had comprehended words at each of the three age points at which the data were collected, and hence the total number of collected vocabularies was 105. For expressive lexicons, the total number of lexicons collected at five different age points was 142 (26 children had no words at 0;9, 6 children had no words at 1;0 and 1 child had no words at $1 ; 3)$. The grouping used for both lexicons was the same, based on earlier studies focusing on the vocabulary composition (Bates et al., 1994; Caselli et al., 1995; Caselli et al., 1999). For the receptive lexicon, the grouping, the numbers of the lexicons in each subgroup, and the age points when the lexicons were gathered, were: 1-20 words 20 lexicons (19 gathered at $0 ; 9,1$ gathered at 1;0); 21-50 words 21 (10 at 0;9, 10 at 1;0, 1 at 1;3); 51-100 words 18 (3 at 0;9, 11 at 1;0, 4 at 1;3); 101-200 words 27 (2 at 0;9, 9 at 1;0, 16 at $1 ; 3) ; 201-300$ words 13 ( 1 at $0 ; 9,4$ at $1 ; 0,8$ at $1 ; 3) ; 301-400$ words 6 (6 at $1 ; 3)$. Respectively, for the expressive lexicon, the numbers of the lexicons in each vocabulary size subgroup, and the age points when the lexicons were gathered, were: 1-20 words 71 lexicons (9 gathered at 0;9, 28 at 1;0, 26 at 1;3, 8 at 1;6); 21-50 words 14 ( 1 at $1 ; 0,6$ at $1 ; 3,7$ at 1;6); 51-100 words 15 (2 at 1;3, 11 at 1;6, 2 at $2 ; 0) ; 101-200$ words 8 (7 at 1;6, 1 at 2;0); 201-300 words 11 ( 2 at 1;6, 9 at 2;0); 301-400 words 10 (10 at 2;0); >400 words 13 (13 at 2;0). Based on the total number of words in the lexicon we calculated the percentage of social terms (i.e., onomatopoetic words, names of people, routine words), common nouns, verbs, adjectives, and grammatical function words (i.e., pronouns, prepositions, question words, quantifiers and connectives) in each lexicon. Then the mean values of the percentages were calculated for each lexicon size subgroup, and the compositions of the lexicons of different sizes were described. The focus of this part of the study was on describing the compositions of the receptive and expressive lexicons of different sizes only, and not, for example, the performance of individual children. No statistical analyses were accomplished based on these percentages. In the present study, the data of the longitudinal sample were analysed using a parallel procedure to that in previous cross-sectional samples (Bates et al., 1994; Caselli et al., 1995; Caselli et al., 1999).

Generalized estimating equations were used to perform repeated-measures Poisson regressions on the analysis of gender differences in vocabulary size. Gender and age were used as independent variables, and lexicon size as dependent variable. If there was a significant age by gender interaction in the repeated-measures analysis, individual age points were also examined separately. When the effect across individual age points was similar, only the result of the repeated-measures analysis was reported. Results were considered significant if the $p$-value was below 0.05 . All significance tests were 2-tailed. 


\section{RESULTS}

\section{Growth of the receptive and expressive lexicon}

The sizes of the receptive and expressive lexicons at each age point are shown in Table 1. All children were reported to have words in their receptive vocabularies already at 0;9. For the expressive lexicon, nine children (26\%) produced words at $0 ; 9,29(83 \%)$ at $1 ; 0$, and 34 children $(97 \%)$ at $1 ; 3$. At 1;6 all children had produced their first word.

The receptive lexicon grew rapidly right from the beginning. At a group level, the acquisition rate accelerated slightly in relation to time: the mean value of acquired new words in the receptive lexicon was 64 (SD 42, median 59) in the 3-month period from $0 ; 9$ to $1 ; 0$, and 94 words (SD 47, median 88) in the period from $1 ; 0$ to $1 ; 3$. However, there was high variation between individual children in the number of acquired new comprehended words in both 3-month intervals: the number of new words varied from 8 to 157 words in the period from $0 ; 9$ to $1 ; 0$, and from 17 to 220 words in the period from $1 ; 0$ to $1 ; 3$. Furthermore, individual children differed in their acquisition styles (Table 2$)$. For most children $(n=26,74 \%)$ the number of acquired new words was higher in the latter 3-month period than in the former one. The lexical acquisition rate of 9 children (26\%) slowed down, however, in the period from 1;0 to 1;3 when compared with the earlier period. Sixteen children (46\%) acquired at least 60 new words during the first 3-month period, and 14 of these children continued to acquire at least the same number of new words also during the second follow-up period. In addition, 12 children (35\%) acquired 60 new words or more for the first time during the period from $1 ; 0$ to $1 ; 3$. The mean

Table 1 Size of the receptive lexicon at 0;9, 1;0,1;3, and the expressive lexicon at $0 ; 9,1 ; 0,1 ; 3,1 ; 6$ and $2 ; 0$

\begin{tabular}{|c|c|c|c|c|c|c|}
\hline \multirow[t]{2}{*}{ Lexicon, age } & \multicolumn{2}{|c|}{ Checklist } & \multicolumn{2}{|c|}{ Extra } & \multicolumn{2}{|c|}{ Combined } \\
\hline & Mean (SD) & Range & Mean (SD) & Range & Mean (SD) & Range \\
\hline \multicolumn{7}{|l|}{ Receptive } \\
\hline $0 ; 9$ & $33(42)$ & $1-205$ & $1(2)$ & $1-8$ & $34(43)$ & $2-212$ \\
\hline $1 ; 0$ & $94(68)$ & $12-262$ & $4(7)$ & $0-33$ & $98(73)$ & $12-271$ \\
\hline $1 ; 3$ & $184(78)$ & $28-333$ & $8(9)$ & $0-41$ & $192(82)$ & 29-343 \\
\hline \multicolumn{7}{|l|}{ Expressive } \\
\hline $0 ; 9$ & $1(2)$ & $0-14$ & $0(0)$ & $0-1$ & $1(3)$ & $0-15$ \\
\hline $1 ; 0$ & $4(6)$ & $0-32$ & $0(1)$ & $0-6$ & $4(7)$ & $0-38$ \\
\hline $1 ; 3$ & $17(18)$ & $0-80$ & $2(3)$ & $0-16$ & $19(20)$ & $0-96$ \\
\hline $1 ; 6$ & 69 (63) & 4-261 & $8(8)$ & $0-39$ & $76(68)$ & $5-275$ \\
\hline $2 ; 0$ & 324 (118) & $60-532$ & $18(13)$ & $0-63$ & 342 (120) & $70-548$ \\
\hline
\end{tabular}


Table 2 The number of new words in receptive and expressive lexicons in each data collection interval for individual children

\begin{tabular}{|c|c|c|c|c|c|c|}
\hline \multirow[t]{2}{*}{ Subject } & \multicolumn{2}{|c|}{ Receptive lexicon } & \multicolumn{4}{|c|}{ Expressive lexicon } \\
\hline & $0 ; 9-1 ; 0$ & $1 ; 0-1 ; 3$ & $0 ; 9-1 ; 0$ & $1 ; 0-1 ; 3$ & $1 ; 3-1 ; 6$ & $1 ; 6-2 ; 0$ \\
\hline 1 & 24 & 83 & 5 & 13 & 56 & 343 \\
\hline 2 & 23 & 120 & 4 & 33 & 66 & 296 \\
\hline 3 & 37 & 100 & 3 & 14 & 78 & 272 \\
\hline 4 & 11 & 19 & 3 & 1 & 3 & 77 \\
\hline 5 & 79 & 114 & 1 & 5 & 16 & 271 \\
\hline 6 & 157 & 56 & 5 & 11 & 4 & 453 \\
\hline 7 & 21 & 140 & 5 & 3 & 8 & 441 \\
\hline 8 & 68 & 87 & 0 & 18 & 32 & 376 \\
\hline 9 & 68 & 88 & 3 & 14 & 44 & 190 \\
\hline 10 & 68 & 87 & 0 & 3 & 4 & 405 \\
\hline 11 & 59 & 50 & 0 & 15 & 66 & 279 \\
\hline 12 & 33 & 134 & 1 & 9 & 4 & 103 \\
\hline 13 & 17 & 37 & 6 & 8 & 7 & 49 \\
\hline 14 & 59 & 45 & 12 & 58 & 205 & 273 \\
\hline 15 & 100 & 119 & 5 & 18 & 68 & 307 \\
\hline 16 & 50 & 108 & 3 & 3 & 51 & 332 \\
\hline 17 & 57 & 65 & 2 & 7 & 30 & 181 \\
\hline 18 & 114 & 137 & 0 & 7 & 130 & 238 \\
\hline 19 & 81 & 220 & 2 & 5 & 142 & 75 \\
\hline 20 & 18 & 88 & 0 & 4 & 12 & 227 \\
\hline 21 & 89 & 79 & 3 & 34 & 94 & 334 \\
\hline 22 & 103 & 91 & 8 & 33 & 117 & 216 \\
\hline 23 & 32 & 54 & 1 & 2 & 2 & 214 \\
\hline 24 & 72 & 38 & 0 & 0 & 21 & 244 \\
\hline 25 & 149 & 71 & 14 & 23 & 193 & 281 \\
\hline 26 & 143 & 86 & 1 & 11 & 31 & 282 \\
\hline 27 & 152 & 75 & 23 & 58 & 82 & 314 \\
\hline 28 & 8 & 17 & 0 & 12 & 1 & 200 \\
\hline 29 & 32 & 95 & 2 & 1 & 89 & 310 \\
\hline 30 & 34 & 102 & 1 & 9 & 54 & 257 \\
\hline 31 & 71 & 139 & 2 & 11 & 40 & 363 \\
\hline 32 & 44 & 124 & 1 & 6 & 31 & 195 \\
\hline 33 & 36 & 40 & 10 & 1 & 61 & 280 \\
\hline 34 & 43 & 180 & 1 & 27 & 167 & 274 \\
\hline 35 & 95 & 195 & 0 & 15 & 12 & 364 \\
\hline
\end{tabular}

Note. Values are combined lexical values (checklist and extra words combined). 
value of the lexicon size for these 28 children at the age point before the strong growth in the acquisition rate occurred for the first time was 44 words (SD 35, median 35, min. 9, max. 114) at 0;9, and 46 words (SD 18, median 49, min. 22, max. 81) at $1 ; 0$.

The expressive lexicon grew slowly at first, but accelerated. At a group level, the mean value of acquired new words was 4 (SD 5, median 2 ) in the period from 0;9 to $1 ; 0,14$ words (SD 14, median 11) in the period from $1 ; 0$ to $1 ; 3,58$ words (SD 56 , median 44) in the period from $1 ; 3$ to $1 ; 6$, and 266 words (SD 96, median 274) in the last 6-month period from $1 ; 6$ to $2 ; 0$. The variation between individual children in the number of acquired new words was not very high at the beginning, but grew in relation to the time: the number of new acquired words varied from 0 to 23 words in the period from $0 ; 9$ to $1 ; 0$, from 0 to 58 words in the period from $1 ; 0$ to $1 ; 3$, from 1 to 205 words in the period from $1 ; 3$ to $1 ; 6$, and from 50 to 454 words in the last 6 -month period. For most children $(n=29,83 \%)$, the acquisition rate of new words was the fastest during the last 6-month follow-up period. There were six children however, whose growth rates slowed down in the latter period when compared with the period from $1 ; 3$ to $1 ; 6$ (Table 2). None of the children acquired 60 words or more during the first two follow-up periods. For most children (32 children, $91 \%$ ) there was a strong growth in the acquisition rate of the expressive lexicon. In the period from $1 ; 3$ to $1 ; 6,14$ children acquired 60 words or more, and from these 13 acquired at least 120 new words during the last 6-month period. In addition, 18 children acquired at least 120 new words for the first time in the last follow-up period. The mean value of the expressive lexicon size at the age point before the strong growth occurred for the first time was 31 words (SD 26, median 27, min. 3, max. 96) at 1;3, and 35 words (SD 21, median 33, min. 5, max. 74) at 1;6.

\section{Effect of gender}

For the receptive lexicon, the interaction between gender and age on the vocabulary growth was not significant, $\chi^{2}(2)=0.7, p=0.72$. Thus, the effect of gender was similar at each age point. Furthermore, the effect of gender alone was not significant, although the values for girls tended to be slightly higher, $\chi^{2}(1)=1.7, p=0.19$. The influence of age was significant, $\chi^{2}(2)=29.8, p<0.0001$ (95\% confidence intervals for estimated mean values: at $0 ; 9$ for girls $22.4-75.9$ and for boys 16.5-43.3; at 1;0 for girls 80.7-156.2 and for boys 60.2-118.5; at 1;3 for girls 184.4-265.5 and for boys 135.9-197.7).

For the expressive lexicon, the effect of the interaction between age and gender on lexicon growth was significant, $\chi^{2}(4)=10.8, p=0.03$. Thus, the effect of gender was different at different age points. Further analysis showed that girls had significantly larger lexicon size at $1 ; 3, \chi^{2}(1)=10.8, p=0.001$, and at $1 ; 6, \chi^{2}(1)=4.20$, $p=0.04$. At other age points studied, girls had larger expressive lexicon sizes, but the difference between sexes was not significant (95\% confidence intervals for estimated mean values: at 0;9 for girls $0.4-4.7$ and for boys $0.1-0.8$; at $1 ; 0$ for girls 2.7-11.7 and for boys 2.0-5.5; at 1;3 for girls 17.7-40.7 and for boys 7.4-15.3; at $1 ; 6$ for girls $71.0-139.5$ and for boys 33.8-87.0; at 2;0 for girls 323.6-418.0 and for boys 264.2-383.8). 


\section{Development of the composition of the receptive and expressive lexicon}

For the receptive lexicon, the numbers of words belonging to different lexical categories at three different age points are presented in Table 3. At a group level, all lexical categories (i.e., social terms, nouns, predicates and function words) were already represented in the receptive lexicons at 0;9. Social terms and nouns were the two largest groups at 0;9 and at 1;0. During the 6-month follow-up period, nouns were acquired most rapidly, but verbs were acquired fast as well. The acquisition rates of adjectives and grammatical functions words were the slowest. At 1;3, the receptive lexicon of the group consisted of $51 \%$ nouns, $17 \%$ verbs, $16 \%$ social terms, $6 \%$ grammatical functions words and $5 \%$ adjectives.

The composition of the receptive lexicons of different sizes is described in Fig. 1. The percentage of social terms was high in small lexicons, but decreased as the vocabulary size grew. The percentage of nouns was increasing in small vocabulary sizes, and started to decrease slightly in the two largest receptive lexicons (i.e., 201-300 and 301-400 words). The percentage of verbs increased from small lexicons to large ones, as did the percentage of adjectives. The percentage of adjectives grew more slowly than that of verbs. The proportion of function words stayed very much the same at all the different lexicon sizes, but the variation in the percentages inside the vocabulary size subgroups decreased as the lexicon size grew. This stabilization happened in the acquisition of all lexical categories.

For the expressive lexicon, the numbers of words belonging in different lexical categories at five different age points are presented in Table 4. At 0;9 and at 1;0,

Table 3 Development of the composition of the receptive lexicon at three age points: means and (in brackets) standard deviations

\begin{tabular}{lccc}
\hline & $0 ; 9$ & $1 ; 0$ & $1 ; 3$ \\
\hline Social terms & $10(7)$ & $20(9)$ & $30(9)$ \\
Onomatopoetic & $2(2)$ & $6(4)$ & $9(4)$ \\
People & $4(2)$ & $6(3)$ & $9(3)$ \\
$\quad$ Routines & $4(4)$ & $8(5)$ & $12(4)$ \\
Common nouns & $14(20)$ & $47(37)$ & $98(42)$ \\
Predicates & $7(14)$ & $21(19)$ & $43(21)$ \\
Verbs & $5(11)$ & $16(14)$ & $32(15)$ \\
Adjectives & $2(4)$ & $5(6)$ & $10(6)$ \\
Function words & $2(3)$ & $6(6)$ & $12(7)$ \\
Pronouns & $0(0)$ & $1(1)$ & $1(2)$ \\
Prepositions & $1(2)$ & $3(3)$ & $6(4)$ \\
Question words & $1(1)$ & $2(1)$ & $3(2)$ \\
Quantifiers & $0(0)$ & $1(1)$ & $2(2)$ \\
\hline
\end{tabular}

Note. Values are combined lexical values (checklist and extra words combined). 


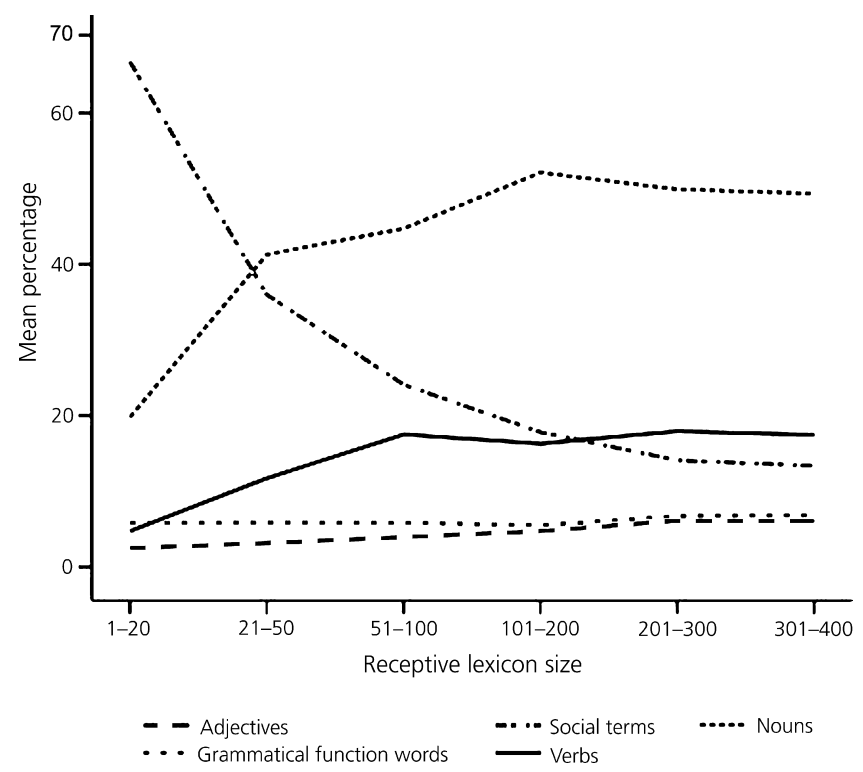

Figure 1 The composition of the receptive lexicon of 35 Finnish children as a function of lexicon size

Table 4 Development of the composition of the expressive lexicon at five age points: means and (in brackets) standard deviations

\begin{tabular}{lccccc}
\hline & $0 ; 9$ & $1 ; 0$ & $1 ; 3$ & $1 ; 6$ & $2 ; 0$ \\
\hline Social terms & $1(2)$ & $3(4)$ & $9(7)$ & $21(10)$ & $44(9)$ \\
$\quad$ Onomatopoetic & $0(1)$ & $2(2)$ & $4(4)$ & $8(4)$ & $13(2)$ \\
People & $0(1)$ & $1(2)$ & $3(2)$ & $8(4)$ & $16(5)$ \\
$\quad$ Routines & $0(1)$ & $0(1)$ & $2(2)$ & $5(4)$ & $16(5)$ \\
Common nouns & $0(0)$ & $1(3)$ & $7(10)$ & $39(44)$ & $169(54)$ \\
Predicates & $0(0)$ & $0(0)$ & $1(2)$ & $9(11)$ & $80(40)$ \\
Verbs & $0(0)$ & $0(0)$ & $1(1)$ & $6(8)$ & $58(29)$ \\
Adjectives & $0(0)$ & $0(0)$ & $0(1)$ & $3(4)$ & $22(12)$ \\
Function words & $0(0)$ & $0(0)$ & $0(1)$ & $3(4)$ & $26(12)$ \\
$\quad$ Pronouns & $0(0)$ & $0(0)$ & $0(0)$ & $1(1)$ & $4(3)$ \\
Prepositions & $0(0)$ & $0(0)$ & $0(0)$ & $2(2)$ & $11(5)$ \\
Question words & $0(0)$ & $0(0)$ & $0(0)$ & $0(1)$ & $4(2)$ \\
Quantifiers & $0(0)$ & $0(0)$ & $0(0)$ & $1(1)$ & $5(3)$ \\
Connectives & $0(0)$ & $0(0)$ & $0(0)$ & $0(0)$ & $2(2)$ \\
\hline
\end{tabular}

Note. Values are combined lexical values (checklist and extra words combined). 
there were only a few words in children's vocabularies. These first lexical items were mostly words belonging to the category of social terms. At 1;3 children had mainly social terms and common nouns in their expressive vocabularies. Furthermore, 17 children (49\%) had verbs in their lexicons at that age, but the numbers of verb types were small (min. 1, max. 3). Only a few children had adjectives or grammatical functions words in their vocabularies (adjectives: 4 children, 11\%; pronouns: 1, 3\%; prepositions and question words: 3, 9\%; quantifiers: $2,6 \%)$ at 1;3, and the numbers of these word types were small (adjectives: min. 1, max. 6; other mentioned word categories: min. 1, max. 2). The numbers of these word types in lexicons had increased at 1;6 however, and the growth of these lexical categories continued in the last part of the second year. At 2;0 the expressive lexicon of the group consisted of $49 \%$ common nouns, $17 \%$ verbs, $13 \%$ social terms, $8 \%$ grammatical function words and $6 \%$ adjectives.

The composition of the expressive lexicons of different sizes is described in Fig. 2. In small lexicons, the percentage of social terms was high, but decreased as the lexicon size grew. The percentage of nouns increased in vocabularies of 1-200 words, and started to decrease after that. The percentage of verbs increased slowly from small lexicons to large ones. The percentages of adjectives and function words grew in a comparable manner, both slower than that of verbs. The variability in the percentages inside the vocabulary size subgroups decreased as the lexicon size grew.

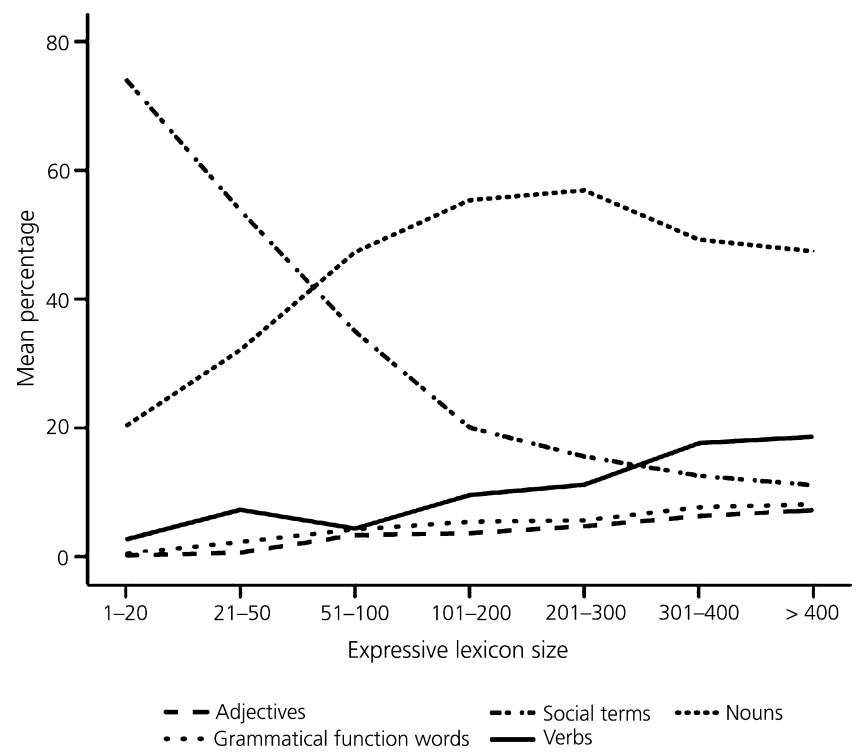

Figure 2 The composition of the expressive lexicon of 35 Finnish children as a function of lexicon size 


\section{Reliability}

Pearson's correlation coefficients between the result of the Reynell Developmental Language Scales III (Edwards et al., 1997; Kortesmaa et al., 2001) and the combined lexicon sizes were calculated to verify the values of maternal ratings. The longitudinal correlation between receptive lexicon size at 1;3 and the receptive score in Reynell's test at 2;0 was $r=0.66, p<0.001$, and between receptive lexicon size at $1 ; 3$ and the total Reynell score at 2;0 it was $r=0.69, p<0$. 001. The simultaneous correlation between expressive lexicon size and the Reynell expressive score at 2;0 was $r=0.77, p=<0.001$, and between expressive lexicon size and the Reynell total score at 2;0 it was $r=0.78, p<0.001$.

\section{DISCUSSION}

The present study provides a comprehensive picture of early lexical acquisition in a longitudinal sample of 35 Finnish children. Findings provided detailed information about the acquisition rates of both expressive and receptive lexicons, and confirmed reported results of earlier and faster growth of the receptive lexicon when compared with that of expressive vocabulary. Gender differences were found in the growth of the expressive lexicon, but not in the growth of receptive vocabulary. The composition analysis showed that although the receptive and expressive lexicons were acquired at different times and rates, the acquisition order of lexical semantic categories was comparable in both lexicons when analysed in relation to lexicon size. The category of common nouns was the largest in all except the smallest lexicons. Verbs were acquired more actively in the receptive than in the expressive lexicons. Growth trajectories of different lexical categories detected in vocabularies of different sizes in this sample of Finnish children were parallel with those presented in the literature involving children acquiring other languages.

Children in the present study acquired their receptive lexicons earlier, at a faster rate and with higher individual variation right from the beginning, when compared with the expressive lexicon. These findings are compatible with earlier results. Benedict (1979) reported that eight children in her sample acquired their comprehension lexicons earlier and at a faster rate than expressive vocabularies. A similar finding was presented in the normative study of the CDI (Fenson et al., 1994), and in the standardization study of the Finnish version of the CDI (Lyytinen, 1999). In both of these latter samples there was also higher individual variation in the receptive lexicon size right from the beginning when compared with that of the expressive lexicon. For example, the standard deviation in the receptive lexicon size was 63 at 1;0 in Lyytinen's (1999) sample, when in the expressive lexicon it was 9 at the same age. The earlier growth of the receptive lexicon emphasizes the fact that language development has already begun when children produce their first words. However, the relationship between the receptive and expressive lexicon, and later receptive and expressive language, is not simple. Bates et al. (1995) found high variation in the expressive lexicon size of those children with large receptive lexicons in their sample. Thus, a large comprehension lexicon does not guarantee that children 
proceed in a comparable manner in their expressive vocabulary acquisition. One reason for this dissociation between early receptive and expressive language may be that comprehension and production skills map onto different aspects of nonlinguistic development, and they are mediated by different neural systems (see the review in Bates et al., 1995). Furthermore, Bates et al. (1988) and Bates et al. (1995) found significant correlations between the receptive/expressive lexicon profiles of the children in the period from $1 ; 1$ to $1 ; 8$, and the comprehension/production profiles in grammatical level in these same children at $2 ; 4$. In the present study a highly significant correlation between receptive lexicon size at 1;3, and the receptive score in Reynell's test at 2;0 was found, suggesting that early receptive lexicon is associated with later receptive grammar. The receptive/expressive profiles of the children were not studied, however. There is a need for further investigations in order to understand fully the relevance of early receptive lexicon to the growth of expressive vocabulary and to the growth of language skills in general.

The growth rate of the expressive lexicon changed from slow to more rapid in the majority of the children. In addition, most children acquired new words so rapidly during the data collection period when the acquisition rate changed that the definition used for spurt in earlier studies could be applied within a widened time frame. For many individuals this fast growth continued for a longer period. Goldfield \& Reznick (1990) presented a parallel finding. In their sample, $72 \%$ of 18 participants evidenced a period of rapid word learning, and this rapid lexical acquisition continued for several months over the 50-word level. It must be emphasized, however, that the longer data collection intervals in the present study do not allow us to determine whether children actually went through a vocabulary spurt (i.e., a rapid change in the acquisition rate during the short period). However, the present study does provide evidence that a change occurs in the growth rate of the expressive lexicon during the second year in most children. Furthermore, there were also children in the present study showing plateaus in their vocabulary acquisition, when the acquisition rate slowed down, in both lexicons. Goldfield \& Reznick (1990) also reported a similar finding in the expressive lexicon. Lastly, fast growth was also detected in the receptive lexicon. Because of the large receptive lexicon sizes already at the early stages of acquisition, the change in the growth rate was not as prominent as in the expressive lexicon. However, this finding does support the view that acceleration also occurs in the receptive lexicon at least for some children.

The lexicon sizes in those children having fast vocabulary growth (i.e., more than 60 words per 3-month period) at the age point before the fast growth happened, were for receptive vocabulary 44 words at 0;9, and 46 words at 1;0, and for expressive vocabulary 31 words at 1;3, and 35 words at 1;6. These vocabulary sizes are interestingly similar, and they suggest that the acceleration in growth may be connected to vocabulary size, as suggested in the definitions of spurt (e.g., Barrett, 1995; Goldfield \& Reznick, 1990). Our data on when the acceleration occurs also suggest that the change in the acquisition rate may be connected to the acquisition of nouns (e.g., Goldfield \& Reznick, 1990).

Those children whose expressive vocabulary acquisition was slower than in others (i.e., the definition of 60 words per 3-months, or 120 per 6-months could not be applied) had the smallest lexicon sizes at 2;0. Nevertheless, they also showed 
growth in their expressive lexicon. It is possible that they went through a short acceleration phase in their expressive lexicon development, but the acquisition rate slowed down. Alternatively, they were in the middle of the accelerating period at 2;0, or went through it later (Goldfield \& Reznick, 1990, 1996; Mervis \& Bertrand, 1995). It must be noted, however, that there was a strong positive correlation between expressive lexicon size and the result of Reynell's test at 2;0. This finding suggests that the expressive lexicon constitutes an important domain of language skills at the end of the second year. In addition, recent studies have also proposed that the growth of the expressive lexicon and emergence of grammar are closely associated at the end of the second year (e.g., Bates \& Goodman, 1997). Thus, it may be that the active growth of the expressive lexicon at the end of the second year, and lexicon size at 2;0, are reflecting the growth of language in a wider manner than usually thought.

There was a trend for girls to have larger lexicon sizes in both their receptive and expressive vocabularies, as has been reported in previous studies (Bornstein et al., 2004; Fenson et al., 1994). In an earlier study (Stolt et al., 2007) we also found that girls had larger lexicons than boys at 2;0. The fact that this difference was not significant in the receptive lexicon in the present study may be due to the relatively small sample size.

When the percentages of the semantic lexical categories were calculated in lexicons of different sizes, a shift from the use of social terms to reference, to predication and to grammar was detected in both lexicons. This shift is in line with the proposal of Bates et al. (1994) and Caselli et al. (1999), who showed comparable growth trajectories for lexical categories in expressive vocabularies in the crosssectional samples of English and Italian children. Similar findings in the expressive lexicon have been reported in children acquiring Spanish (Jackson-Maldonado et al., 1993) and Hebrew (Maital et al., 2000; cf. Bornstein et al., 2004; Kauschke \& Hofmeister, 2002). The fact that parallel growth trajectories are detected in children acquiring different target languages suggests that the acquisition of lexical semantic categories is independent of the structure of the target language. Furthermore, the finding that lexical categories were acquired in a parallel manner in both lexicons is also compatible with findings reported previously. Caselli et al. (1995) presented parallel growth trajectories in both the receptive as well as in the expressive lexicons of English and Italian children. Benedict (1979) reported that the growth order of lexical categories was comparable in both the receptive and expressive vocabularies (i.e., the first 50 words in both lexicons). This overall symmetry is interesting, especially when taking into consideration that the two lexicons are acquired at different times and rates. However, in both studies (Benedict, 1979; Caselli et al., 1995) it was also found that verbs were acquired more actively in the receptive than in the expressive lexicons, a phenomenon also detected in the present study. But why are verbs acquired earlier in comprehended than in expressed lexicons? Goldfield (2000) hypothesized that one explanation for this may be in pragmatics. In early interactions analysed by Goldfield (2000) mothers more often prompted children to produce an action than produce a verb. Mothers also elicited children's noun production, but only rarely asked them to produce verbs. Thus, verb comprehension was favoured over production. 
In our previous study of another cross-sectional sample of 84 Finnish children at 2;0, comparable growth trajectories in the expressive lexicon were found (Stolt et al., 2007). The finding that parallel trajectories for lexical categories are detected in a cross-sectional sample at 2;0 and in the present longitudinal one in children aged between 0;9 and 2;0 suggests that growth of lexical semantic categories are related to vocabulary growth during the second year and at the age of 2;0, and not necessarily to the chronological age of the child. Bates \& Goodman (1997) reported that the expressive lexicon size predicted grammatical growth better than age did at the end of the second year. Our findings in two independent samples gathered at different age points also suggest that the development of early lexical composition is tied more to vocabulary growth than to the age of child.

The correlations between the values of the structured maternal ratings and the result of the formal language test were strong and significant. Furthermore, the checklist values of the present study and those presented by Lyytinen (1999) are highly comparable. The checklist value for the expressive lexicon size at 2;0 was slightly higher than the one in our earlier study (Stolt et al., 2007). This difference may be because all children in the present sample were firstborns, whereas the sample in our previous study was randomized. The high correlations between the results of the maternal rating method and that of the formal language test, as well as parallel results from different studies, further support the clinical use of the structured parental rating method when evaluating the lexical growth of very young children. These comparable findings in different samples also confirm general outlines for the growth rate of the lexicon in children acquiring Finnish (see also Kunnari, 2000).

The present study showed highly comparable patterns in early lexical acquisition in the sample of 35 Finnish children as described in the literature in children acquiring other languages. The receptive lexicon was acquired earlier, at a faster rate and with higher individual variation right from the beginning, as compared with the expressive lexicon. The growth order and the growth trajectories of lexical semantic categories were compatible with those described in children acquiring English, Italian, Spanish and Hebrew. These findings suggest that the growth order and the growth rates of receptive and expressive vocabularies and the acquisition of the lexical semantic categories are acquired in a highly comparable manner, irrespective of the structure of the target language.

\section{ACKNOWLEDGEMENTS}

This study was supported by grants from the Emil Aaltonen Foundation, the Friends of Paediatric Clinics in University Hospitals in Finland Fund, the Neonatal Research in Southwestern Finland Fund, The Finnish Cultural Foundation, and by Langnet, the national postgraduate school in language studies in Finland, and Academy of Finland.

We thank Jaakko Matomäki for help in the data analyses. We are grateful to all the families for participating in the study. This study is part of the PIPARI study; the study group comprises: Mikael Ekblad, Satu Ekblad, Eeva Ekholm, Leena Haataja, Pentti Kero, Jarkko Kirjavainen, Riikka Korja, Harry Kujari, Helena Lapinleimu, Liisa Lehtonen, Hanna Manninen, Jaakko Matomäki, Jonna Maunu, Petriina Munck, Pekka Niemi, Pertti Palo, Riitta Parkkola, Jorma Piha, Annika Pihlgren, Liisi Rautava, Päivi Rautava, Milla Reiman, Hellevi Rikalainen, Katriina Saarinen, Elina 
Savonlahti, Matti Sillanpää, Suvi Stolt, Päivi Tuomikoski-Koiranen, Anu Uschakoff and Tuula Äärimaa.

\section{REFERENCES}

Barrett, M. (1995). Early lexical development. In P. Fletcher \& B. MacWhinney (Eds), The handbook of child language (pp. 96-151). Oxford: Blackwell.

Bassano, D., Eme, P.-E. \& Champaud, C. (2005). A naturalistic study of early lexical development: General processes and inter-individual variations in French children. First Language, 25, 67-101.

Bates, E., Bretherton, I. \& Snyder, L. (1988). From first words to grammar. Individual differences and dissociable mechanisms. New York: Cambridge University Press.

Bates, E., Dale, P. \& Thal, D. (1995). Individual differences and their implications for theories of language development. In P. Fletcher \& B. MacWhinney (Eds), The handbook of child language (pp. 96-151). Oxford: Blackwell.

Bates, E. \& Goodman, J. (1997). On the inseparability of grammar and the lexicon: Evidence from acquisition, aphasia and real-time processing. Language and cognitive processing, 12, 507-584.

Bates, E., Marchman, V., Thal, D., Fenson, L., Dale, P., Reznick, S., Reilly, J. \& Hartung, J. (1994). Developmental and stylistic variation in the composition of early vocabulary. Journal of Child Language, 21, 85-123.

Benedict, H. (1979). Early lexical development: Comprehension and production. Journal of Child Language, 6, 183-200.

Bornstein, M., Cote, L., Maital, S., Painter, K., Park, S.-Y., Pascual, L., et al. (2004). Cross-linguistic analysis of vocabulary in young children: Spanish, Dutch, French, Hebrew, Italian, Korean and American English. Child Development, 75, 1115-1139.

Bornstein, M., Haynes, M. \& Painter, K. (1998). Sources of child vocabulary competence: A multivariate model. Journal of Child Language, 25, 367-393.

Bornstein, M., Leach, D. \& Haynes, O. (2004). Vocabulary competence in first- and second-born siblings of the same chronological age. Journal of Child Language, 31, 855-873.

Bornstein, M. H., Putnick, D. L. \& De Houwer, A. (2006). Child vocabulary across the second year: Stability and continuity for reporter comparisons and a cumulative score. First Language, 26, 299-316.

Caselli, M., Bates, E., Casadio, P., Fenson, J., Fenson, L., Sanderl, J. \& Weir, J. (1995). A cross-linguistic study of early lexical development. Cognitive Development, 10, 159-199.

Caselli, M., Casadio, P. \& Bates, E. (1999). A comparison of the transition from first words to grammar in English and Italian. Journal of Child Language, 26, 69-111.

Dasinger, L. (1997). Issues in the acquisition of Estonian, Finnish, and Hungarian: A crosslinguistic comparison. In D. Slobin (Ed.), The crosslinguistic study of language acquisition. Vol. 4 (pp. 87-182). Mahwah, NJ: Erlbaum.

D'Odorico, L., Carubbi, S., Salerni, N. \& Calvo, V. (2001). Vocabulary development in Italian children: A longitudinal evaluation of quantitative and qualitative aspects. Journal of Child Language, 28, 351-372.

Dromi, E. (1999). Early lexical development. In M. Barret (Ed.), The development of language: Studies in experimental psychology (pp. 99-131). London: Psychology Press Ltd.

Edwards, S., Fletcher, P., Garman, M., Hughes, A., Letts, C. \& Sinka, I. (1997). Reynell developmental language scales. Third edition manual. Berkshire, UK: The NFER Nelson Publishing Co. Ltd. 
Eriksson, M. \& Berglund, E. (1999). Swedish early communicative development inventories: Words and gestures. First Language, 19, 55-90.

Fenson, L., Dale, P., Reznick, J., Bates, E., Thal, D. \& Pethick, S. (1994). Variability in early communicative development. Monographs of the Society for Research in Child Development, 59(5, Serial No. 242).

Ganger, J. \& Brent, M. (2004). Re-examining the vocabulary spurt. Developmental Psychology, 40(4), 621-632.

Gentner, D. (1982). Why nouns are learned before verbs: Linguistic relativity versus natural partitioning. In M. Bowerman \& S. C. Levinson (Eds), Language acquisition and conceptual development (pp. 301-334). New York: Cambridge University Press.

Gentner, D. \& Boroditsky, L. (2001). Individuation, relativity, and early word learning. In M. Bowerman \& S. Levinson (Eds), Language acquisition and conceptual development (pp. 215-256). Cambridge: Cambridge University Press.

Goldfield, B. (2000). Nouns before verbs in comprehension vs. production: the view from pragmatics. Journal of Child Language, 27, 501-520.

Goldfield, B. \& Reznick, S. (1990). Early lexical acquisition: Rate, content, and the vocabulary spurt. Journal of Child Language, 17, 171-183.

Goldfield, B. \& Reznick, S. (1996). Measuring the vocabulary spurt: A reply to Mervis \& Bertrand. Journal of Child Language, 23, 241-246.

Harris, M., Yeeles, C., Chasin, J. \& Oakley, Y. (1995). Symmetries and asymmetries in early lexical comprehension and production. Journal of Child Language, 22, 1-18.

Jackson-Maldonado, D., Thal, D., Marchman, V., Bates, E. \& Gutierrez-Clellen, V. (1993). Early lexical development in Spanish-speaking infants and toddlers. Journal of Child Language, 20, 523-549.

Kauschke, C. \& Hofmeister, C. (2002). Early lexical development in German: A study on vocabulary growth and vocabulary composition during the second and third year of life. Journal of Child Language, 29, 735-757.

Kern, S. (2007). Lexicon development in French-speaking infants. First Language, 27, 227-250.

Kortesmaa, K., Merikoski, H., Warma, M.-L. \& Varpela, V. (2001). Reynellin kielellisen kehityksen testi [Reynell developmental language scales]. Helsinki: Psykologien kustannus Oy.

Kunnari, S. (2000). Characteristics of early lexical and phonological development in children acquiring Finnish. Doctoral dissertation, University of Oulu.

Lyytinen, P. (1999). Varhaisen kommunikaation ja kielen kehityksen arviointimenetelmä [A methed to assess early communication and language development]. Jyväskylä: The Child Research Center of the University of Jyväskylä and Niilo Mäki institute.

Lyytinen, P. \& Lyytinen, H. (2004). Growth and predictive relations of vocabulary and inflectional morphology in children with and without familial risk for dyslexia. Applied Psycholinguistics, 25, 397-411.

Maital, S., Dromi, E., Sagi, A. \& Bornstein, M. (2000). The Hebrew communicative development inventory: Language specific properties and cross-linguistic generalizations. Journal of Child Language, 27, 43-67.

Menyuk, P., Liebergott, J. \& Schultz, M. (1995). Early language development in full-term and in premature infants. Hillsdale, NJ: Erlbaum.

Mervis, C. \& Bertrand, J. (1995). Early lexical acquisition and the vocabulary spurt: A response to Goldfield \& Reznick. Journal of Child Language, 22, 461-468.

Nieminen, P. (1991). Äidin ja lapsen kommunikaatio ja lapsen kielen omaksuminen [The communication of a mother and a child, and the language acquisition of child]. Doctoral dissertation, University of Tampere. 
Reznick, S. \& Goldfield, B. (1992). Rapid change in lexical development in comprehension and production. Developmental Psychology, 28, 406-413.

Ring, E., \& Fenson, L. (2000). The correspondence between parent report and child performance for receptive and expressive vocabulary beyond infancy. First Language, 20, 141-159.

Robinson, B. \& Mervis, C. (1999). Comparing productive vocabulary measures from the CDI and a systematic diary study. Journal of Child Language, 26, 177-185.

Salerni, N., Assanelli, A., D'Odorico, L. \& Rossi, G. (2007). Qualitative aspects of productive vocabulary at the 200- and 500-word stages: A comparison between spontaneous speech and parental report data. First Language, 27, 75-87.

Stolt, S., Klippi, A., Launonen, K., Munck, P., Lehtonen, L., Lapinleimu, H., Haataja, L. \& the PIPARI study group (2007). Size and composition of the lexicon in prematurely born very-low-birth-weight and full-term Finnish children at two years of age. Journal of Child Language, 34, 283-310.

Szagun, G., Steinbrink, C., Franik, M. \& Stumper, B. (2006). Development of vocabulary and grammar in young German-speaking children assessed with a German language development inventory. First Language, 26, 259-280.

Thordardottir, E. \& Weismer, S. (1996). Language assessment via parent report:

Development of a screening instrument for Icelandic children. First Language, 16, 265-285.

Tomasello, M. (2003). Constructing a language. A usage-based theory of language acquisition. Cambridge, MA, \& London: Harvard University Press.

\section{ADDRESS FOR CORRESPONDENCE}

Suvi Stolt

University of Turku, School of Finnish and General Linguistics / Logopedics, Itäinen Pitkäkatu 3a, 20014 Turku, Finland

E: Suvi.Stolt@utu.fi 